\title{
Síntomas de depresión y ansiedad en jóvenes universitarios: prevalencia y factores relacionados
}

\author{
Katherine M. Arrieta Vergaraa ${ }^{a}$ Shyrley Díaz Cárdenas ${ }^{a}$ y Farith González Martínezº .
}

\begin{abstract}
a Odontóloga. Magíster en Salud Pública. Departamento de Odontología Preventiva y Social. Facultad de Odontología. Universidad de Cartagena. Cartagena de Indias (Colombia).

${ }^{b}$ Odontólogo. Magíster en Salud Pública. Departamento de Investigaciones. Facultad de Odontología. Universidad de Cartagena. Cartagena de Indias (Colombia).
\end{abstract}

\section{Correspondencia:}

Katherine M. Arrieta Vergara. Departamento de Odontología Preventiva y Social.

Facultad de Odontología.

Campus de la Salud.

Universidad de Cartagena.

Barrio Zaragocilla.

Cartagena de Indias

(Colombia).

Correo electrónico:

karrietav@unicartagena.edu.co

Recibido el 9 de octubre de 2013.

Aceptado para su publicación el 12 de enero de 2014.

\section{RESUMEN}

Objetivo: Estimar la asociación entre síntomas depresivos y ansiosos con factores sociodemográficos, académicos, conductuales y familiares entre estudiantes universitarios de Cartagena, Colombia.

Diseño del estudio: Estudio transversal.

Emplazamiento: Universidad pública de Cartagena, Colombia.

Participantes: 973 estudiantes universitarios de Cartagena (Colombia), seleccionados a través de un muestreo probabilístico.

Mediciones principales: Se utilizó un instrumento anónimo de auto-reporte que incluyó: variables sociodemográficas (sexo, edad, estrato socioeconómico), el cuestionario de cribado para ansiedad y depresión, cuestionario Goldberg (E.A.D.G), el APGAR familiar para determinar la función familiar y otras preguntas que indagaban por factores asociados. Los datos fueron analizados a partir de frecuencias y proporciones, razones de disparidad y regresión logística nominal.

Resultados: La prevalencia de síntomas ansiosos y depresivos fue del $76,2 \%$ y $74,4 \%$ respectivamente. Los factores asociados con síntomas ansiosos fueron: dificultades económicas, problemas familiares y consumo de alcohol; los relacionados con síntomas depresivos fueron: problemas familiares, dificultades económicas, ansiedad y antecedentes familiares de ansiedad o depresión.

Conclusiones: La sintomatología de ansiedad y depresión fue alta en los universitarios, siendo las dificultades económicas y los problemas familiares los factores que más intervinieron para su aparición. Por lo tanto son necesarias las intervenciones que modifiquen estos comportamientos en beneficio de la calidad de vida de estos jóvenes.

Palabras clave: Depresión. Ansiedad. Estudiantes. Factores de Riesgo.

\section{ABSTRACT}

Depression and anxiety symptoms in young university students: prevalence and related factors.

Objective: To estimate the association between depression and anxiety symptoms and sociodemographic, academic, behavioural and family factors among university students.

Design of the study: Cross-sectional study.

Sample setting: Public University of Cartagena, Colombia.

Participants: 973 university students of Cartagena (Colombia), selected by probability sampling. Main measures: An anonymous self-reporting instrument was used. It included: sociodemographic variables (sex, age, socio-economic status), the screening questionnaire for depression and anxiety, the Goldberg questionnaire (GDAS)

the APGAR family test to determine the family function and other test items which asked about associated factors. The data obtained were analyzed using frequencies and proportions, disparity ratios and nominal logistic regression.

Results: The prevalence of the depression and anxiety symptoms was $76.2 \%$ and $74.4 \%$ respectively. The factors associated to anxiety symptoms were: economic difficulties, family problems and alcohol consumption; the factors related to depression symptoms were: family problems, economic difficulties, anxiety and family history of anxiety or depression.

Conclusions: Anxiety and depression symptomatology was high among university students, being economic difficulties and family problems the most frequent factors causin its onset. Therefore, specific interventions to modify these behaviours for the benefit of the quality of life of these young people are required.

Keywords: Depression. Anxiety. Students. Risk Factors. 


\section{INTRODUCCIÓN}

Los trastornos mentales constituyen una prioridad en salud pública, dado que se encuentran entre las causas más importantes de morbilidad en Atención Primaria ${ }^{1}$. Investigaciones en el campo de la salud mental han fijado su atención en la población universitaria, y diversos estudios señalan que los trastornos depresivos y ansiosos en estudiantes universitarios presentan una mayor tasa en comparación con la población general ${ }^{2}$. Este aumento se debe a que en el ambiente universitario se ponen a prueba competencias y habilidades para alcanzar los objetivos durante el periodo de formación profesional, sometiendo continuamente al estudiante a una gran carga de exigencias académicas, nuevas responsabilidades, evaluaciones, realización de trabajos, algunas veces desarraigo, así como presiones familiares, económicas y sociales, que al interactuar con factores identificados como de riesgo para la presencia de ansiedad y depresión (como el sexo, antecedentes familiares y personales de depresión, diagnóstico de enfermedad grave, muerte de un ser querido, separación de los padres y el consumo de alcohol), pudieran causar eventualmente un deterioro en su salud mental ${ }^{3,4}$.

A todo esto se suma el hecho de que muchos estudiantes de pregrado se encuentran en la etapa de adolescencia tardía, fase de cambios físicos, psicológicos, socioculturales y cognitivos que demandan del joven estrategias de afrontamiento, que le permitan consolidar su identidad, autonomía y éxito personal y social5; esta fase es importante para el logro de comportamientos saludables, que disminuyan riesgos y prevengan el desarrollo de trastornos clínicos durante la adolescencia y en el transcurso de la adulte $z^{4}$. Sobre el particular se ha encontrado que individuos con historias de depresión en la adolescencia presentan un alto porcentaje de continuidad del trastorno depresivo mayor en la edad adulta ${ }^{6}$. Aalton-Setala et al. realizaron un estudio que evaluaba las conclusiones de investigaciones previas acerca de la continuidad de los trastornos afectivos en los adolescentes, indicando cuáles síntomas depresivos reportados en esta población predecían un mayor riesgo de trastornos psiquiátricos y consumo de alcohol en la adultez temprana ${ }^{7}$. Comparado con los sujetos que no reportaban síntomas depresivos en la adolescencia, el riesgo de presentar un trastorno depresivo en la adultez temprana (trastorno depresivo mayor o distimia) o problemas psicosociales fue tres veces mayor para los que sí los habían reportado; de igual forma, se asoció con una mayor ocurrencia de hospitalizaciones y problemas de ajuste social e interpersonales, suicidio e insatisfacción con la vida ${ }^{7}$.

Datos del Segundo Estudio Nacional de Salud y Consumo de Sustancias Psicoactivas muestran una prevalencia de depresión general en Colombia del $25,1 \%$; con respecto a la depresión leve, moderada y severa, sugiere que los jóvenes colombianos de entre 16 y 21 años muestran los más altos índices de depresión en comparación con otros de rangos de edad diferentes, a excepción de la depresión severa en los hombres en el rango de 28 a 33 años$^{8}$. Además, según la secretaría de Bogotá, en el año 2000 la tercera causa de muerte en jóvenes de 14 a 26 años se debió a lesiones autoinfligidas intencionalmente (suicidios) con un $7 \%$ del total de las muertes después de las agresiones violentas y los accidentes de tránsito ${ }^{4}$.

El identificar problemas de salud mental y factores de riesgo en población universitaria es de vital importancia para el desarrollo de su educación integral, formación como individuos y como futuros profesionales, implicando un gran reto dentro de las instituciones universitarias la identificación e intervención de estas problemáticas en búsqueda de un mejora de la calidad de vida de los estudiantes y de los profesionales en formación.

Por todo lo anterior, el propósito de este estudio fue estimar la asociación entre síntomas depresivos y ansiosos con factores socio-demográficos, académicos, conductuales y familiares entre estudiantes universitarios de Cartagena (Colombia).

\section{SUJETOS Y MÉTODOS}

Estudio analítico de corte transversal, realizado en una universidad pública de la ciudad de Cartagena (Colombia). El universo de estudio estuvo conformado por 8.740 estudiantes, seleccionándose 973 a través de un muestreo probabilístico a fijación proporcional por programa académico y nivel cursado. En cada nivel se realizó un muestreo aleatorio simple con reemplazo al interior de los mismos, teniendo en cuenta el listado de asistentes al aula de clase en el momento del muestreo, de acuerdo a un listado aleatorio obtenido por computador. Cada sujeto elegible dentro de cada programa y nivel cursado tuvo la misma probabilidad de hacer parte de la muestra de estudio, y esta probabilidad fue diferente cuando estuvo relacionada con el tamaño de cada programa y de cada nivel en el que se encontró matriculado el estudiante. Para el cálculo del tamaño de la muestra se utilizó el programa $\mathrm{EPI}-\mathrm{INFO}{ }^{\circledR}$, versión 3.5.1, con una con- 
fianza del 95\%, error relativo del 3\% y frecuencia esperada para el evento de trastornos de ansiedad o depresión del $65 \%{ }^{9}$.

El estudio requirió que los participantes estuvieran matriculados en cualquiera de los programas diurnos y presenciales de la universidad en el segundo periodo del año 2012. Además, se pidió su consentimiento informado por escrito para el uso de la información con fines científicos y demás aspectos soportados por las disposiciones internacionales: declaración de Helsinki (modificación de Edimburgo 2000) y la normatividad para Colombia (Resolución 8430 de 1993 del Ministerio de la Salud).

Para la recolección de la información, se aplicó un cuestionario ad hoc de auto-reporte anónimo que contenía 58 preguntas de selección múltiple politómicas con única respuesta, diseñado desde la teoría y evaluada su validez de apariencia por tres jueces expertos, teniendo en cuenta su pertinencia, suficiencia de las categorías, plausibilidad, semántica, sintaxis y ordenamiento de los ítems. De igual forma, fue valorado a través de una prueba piloto en una población con similares características. Un primer instrumento contenía la siguiente información para el estudio de las posibles variables independientes con sus respectivas categorías: características socio-demográficas: edad (adolescentes: $\leq 19$ años; adulto joven: >20 años), sexo, estrato socioeconómico, tipo de programa, nivel o ciclo académico, estado civil, tenencia de hijos, procedencia y ocupación. De igual forma, se evaluaron otros aspectos relacionados con el rol del estudiante al interior de la institución universitaria como rendimiento académico acumulado (deficiente: calificación <3,0; aceptable: calificación entre 3,0 y 3,8; bueno: calificación >3,8), falta de tiempo para descansar (sí o no), dificultades económicas (presencia de escasos ingresos económicos para el sostenimiento durante sus estudios: sí o no) y problemas en las relaciones con amigos, pareja o cónyuge (sí o no), antecedentes personales de tratamientos psicológicos o psiquiátricos (sí o no), antecedentes familiares de ansiedad y depresión (sí o no). Para el consumo actual de alcohol y consumo de riesgo, perjudicial o dependencia al alcohol se utilizó la escala Alcohol Use Disorders Identification Test (AUDIT: punto de corte 7 y 8 para mujeres y hombres respectivamente $)^{10,11}$. Así mismo, para el estudio de los factores familiares fue usada la escala APGAR para la valoración de la función familiar (la cual consta de cinco preguntas que se califican de 0 a 2: "casi siempre", 2 puntos; "a veces", 1 punto; "casi nunca", 0; las puntaciones del APGAR se encuentran entre 0 y 10 puntos; puntuaciones entre 7 y 10 sugieren funcionalidad familiar; e inferiores a 7, disfuncionalidad familiar) y el apoyo de amigos ${ }^{12}$.

Para la medición de las variables dependientes o de resultado (ansiedad y depresión) se utilizó el cuestionario de Goldberg o E.A.D.G ${ }^{13}$ que consta de una escala de ansiedad y otra para la depresión, totalmente independientes; sus elevados valores de sensibilidad y especificidad ${ }^{13}$, han sido la gran motivación para que esta escala haya sido utilizada como instrumento de cribado, tanto con fines asistenciales como epidemiológicos, y como guía de la entrevista clínica en el ámbito de la Atención Primaria. Cada una de las sub-escalas se estructuran en 4 ítems iniciales de despistaje para determinar si es o no probable que exista un trastorno mental, y un segundo grupo de 5 ítems que se formulan sólo si se obtienen respuestas positivas a las preguntas de despistaje (2 o más en la subescala de ansiedad, 1 o más en la sub-escala de depresión), siendo los puntos de corte $\geq 4$ para la escala de ansiedad, y $\geq 2$ para la de depresión ${ }^{13}$. La consistencia interna (alfa de Cronbach) de la escala de ansiedad y depresión Goldberg (EADG) fue de 0,$86 ; 0,81$ para la sub-escala de ansiedad y 0,77 para la de depresión.

El análisis de la información se realizó con ayuda del programa para Windows STATA® 12.0 (Stata Corp LP, College Station, TX, EE.UU.), teniendo en cuenta los factores de expansión correspondientes al diseño muestral utilizando la rutina "syv". Los datos fueron analizados a partir de estadística descriptiva, con el uso de medias, desviaciones estándar, frecuencias absolutas y relativas. Se estimaron por proporciones las ocurrencias de síntomas ansiosos y depresivos, al igual que los factores sociodemográficos, académicos, conductuales, familiares y sociales. Para el análisis bivariado se utilizó la prueba $c^{2}$ de Pearson, y como medida de asociación la razón de disparidad (Odds Ratio), con intervalos de confianza del 95\%. Para efectos descriptivos de conocer la probabilidad o riesgo de sufrir un trastorno depresivo o ansioso, se realizó un análisis múltiple de regresión logística nominal, con la inclusión en los modelos de las variables que mostraron probabilidades menores a 0,10 ; se conformaron modelos a partir de la exclusión de cada una de las variables en un proceso de pasos hacia atrás según las recomendaciones de Greenland ${ }^{14}$.

\section{RESULTADOS}

En el presente estudio participaron 973 estudiantes universitarios con edades comprendidas entre 


\begin{tabular}{|c|c|c|}
\hline Variables sociodemográficas & Frecuencia $(n=973)$ & Porcentaje (\%) \\
\hline $\begin{array}{l}\text { Sexo } \\
\text { Femenino } \\
\text { Masculino }\end{array}$ & $\begin{array}{l}580 \\
393\end{array}$ & $\begin{array}{l}59,61 \\
40,39\end{array}$ \\
\hline $\begin{array}{l}\text { Edad (ciclo vital) } \\
\text { Adolescentes (18 - } 19 \text { años) } \\
\text { Adultos jóvenes (20 - } 35 \text { años) }\end{array}$ & $\begin{array}{l}359 \\
614\end{array}$ & $\begin{array}{l}36,9 \\
63,1\end{array}$ \\
\hline $\begin{array}{l}\text { Estrato } \\
1 \text { (bajo-bajo) } \\
2 \text { (bajo) } \\
3 \text { (medio-bajo) } \\
4 \text { (medio) } \\
5 \text { (medio-alto) }\end{array}$ & $\begin{array}{c}189 \\
366 \\
322 \\
71 \\
25\end{array}$ & $\begin{array}{c}19,42 \\
37,62 \\
33,09 \\
7,3 \\
2,57\end{array}$ \\
\hline $\begin{array}{l}\text { Estado civil } \\
\text { Soltero } \\
\text { Casado } \\
\text { Unión libre }\end{array}$ & $\begin{array}{c}930 \\
19 \\
18\end{array}$ & $\begin{array}{c}95,58 \\
1,95 \\
1,85\end{array}$ \\
\hline $\begin{array}{l}\text { Procedencia } \\
\text { Rural } \\
\text { Urbana }\end{array}$ & $\begin{array}{l}823 \\
150\end{array}$ & $\begin{array}{l}84,58 \\
15,42\end{array}$ \\
\hline $\begin{array}{l}\text { Tienen hijos } \\
\text { Sí } \\
\text { No }\end{array}$ & $\begin{array}{c}39 \\
934\end{array}$ & $\begin{array}{c}4,01 \\
95,99\end{array}$ \\
\hline $\begin{array}{l}\text { Ocupación } \\
\text { Estudia } \\
\text { Estudia y trabaja }\end{array}$ & $\begin{array}{c}875 \\
94\end{array}$ & $\begin{array}{c}89,93 \\
9,66\end{array}$ \\
\hline $\begin{array}{l}\text { Semestre } \\
1 \\
2 \\
3 \\
4 \\
5 \\
6 \\
7 \\
8 \\
9 \\
10\end{array}$ & $\begin{array}{c}59 \\
118 \\
194 \\
145 \\
97 \\
87 \\
78 \\
99 \\
69 \\
27\end{array}$ & $\begin{array}{c}6,06 \\
12,13 \\
19,94 \\
14,90 \\
9,97 \\
8,94 \\
8,02 \\
10,17 \\
7,09 \\
2,77\end{array}$ \\
\hline
\end{tabular}

Tabla 1. Distribución de las variables sociodemográficas entre los estudiantes universitarios de Cartagena (Colombia), 2012

\begin{tabular}{lccc}
\hline Variables & $\begin{array}{c}\text { Frecuencia } \\
(\mathbf{n = 9 7 3 )}\end{array}$ & $\begin{array}{c}\text { Porcentaje } \\
(\%)\end{array}$ & IC95\% \\
\hline Sintomatología de ansiedad & & & \\
Sí $(\geq 4$ puntos) & 741 & 76,1 & $73,5-78,9$ \\
No (<4 puntos) & 232 & 23,8 & $21,0-26,4$ \\
Sintomatología de depresión & & & \\
Sí ( $\geq 2$ puntos) & 734 & 75,4 & $72,7-78,1$ \\
No (<2 puntos) & 239 & 24,5 & $21,8-27,2$ \\
\hline
\end{tabular}

Tabla 2. Prevalencia de sintomatología de ansiedad y depresión según la escala de Goldberg (EADG) entre los estudiantes universitarios de Cartagena (Colombia), 2012 
18 y 35 años, con un promedio de edad 21 años $(\mathrm{DE}=2,6)$. Las características sociodemográficas de los participantes se observan en el tabla 1.

A partir de la escala de Goldberg (EADG), se encontró que el $76,2 \%$ de los estudiantes posiblemente presentan sintomatología de trastornos de ansiedad, y el $75,4 \%$ pueden presentar sintomatología depresiva (tabla 2).

\section{Factores relacionados}

- Académicos: Al evaluar el contexto académico logrado por los estudiantes durante el transcurso de su carrera, el $86,1 \%$ se encuentra con un promedio académico de 3,0 a 3,9 (promedio Aceptable); el 40,4\% no cuentan con suficiente tiempo para descansar; el 92,6\% refirió tener una buena relación con sus compañeros; y el $48 \%$ presentan dificultades económicas.

- Familiares: El $63,9 \%$ de los estudiantes presentaban familias disfuncionales según APGAR familiar, y solo un $7,4 \%$ de los sujetos no contó con soporte de amigos.

- Conductuales: El 47,7\% manifestó presentar problemas familiares, el 33,1\% reportó problemas con la pareja, el $41,2 \%$ ha tenido familiares con antecedentes de ansiedad o depresión y el $11,7 \%$ de los estudiantes se encuentra recibiendo tratamiento psicológico o psiquiátrico. Frente al consumo de alcohol, se encontró que el $65,1 \%$ son consumidores actuales; el consumo de riesgo, perjudicial o riesgo de dependencia de alcohol con la escala AUDIT fue del 18,4\%, donde el $13 \%$ correspondió a consumo perjudicial y el $5,4 \%$ a riesgo de dependencia de alcohol.

\section{Factores asociados a la sintomatología de ansiedad}

La tabla 3 muestra el análisis bivariable y el modelo de regresión logística nominal entre la sintomatología de ansiedad y diversos factores. Con estimadores crudos, se observaron asociaciones estadísticamente significativas entre la sintomatología de ansiedad y las dificultades económicas, presencia de problemas familiares y el consumo actual de alcohol. El modelo que mejor explica la presencia de sintomatología de ansiedad fue el conformado por los siguientes factores: dificultades económicas y consumo actual de alcohol $\left(c^{2}=27,1 ; p=0,000\right)$.

Factores asociados a la sintomatología de depresión
En la tabla 4 se presenta el análisis bivariable y el modelo de regresión logística nominal entre la sintomatología de depresión y diversos factores. Al asociar la presencia de sintomatología para trastornos depresivos con los factores estudiados, se observaron asociaciones estadísticamente significativas para las siguientes variables: dificultades económicas, problemas familiares, antecedentes familiares de ansiedad o depresión y presencia de trastorno de ansiedad. El modelo que mejor explica la presencia de sintomatología de depresión fue el conformado por los siguientes factores: dificultades económicas, antecedentes familiares de ansiedad o depresión y trastornos de ansiedad $\left(\mathrm{C}^{2}=\right.$ 254,6; $p=0,000$ ).

\section{DISCUSIÓN}

Los hallazgos de esta investigación muestran una alta prevalencia de síntomas de ansiedad y depresión en jóvenes universitarios de Cartagena, incluso mucho mayor que la reportada por estudios nacionales e internacionales ${ }^{9,15-17}$. Sin embargo, esto no es una evidencia de diagnóstico formal de ansiedad y depresión, pero sí sugiere que un nivel significativo de estudiantes muestran niveles patológicos o distrés psicológico. Además, cabe aclarar que las diferencias entre las prevalencias reportadas por los estudios no son del todo comparables, ya que utilizan diferentes instrumentos de cribado para ello. Estas cifras diferentes comparadas con otros países pueden deberse al contexto socioeconómico y político colombiano, caracterizado por altos índices de desempleo, violencia y pobreza, incertidumbre laboral y pocas expectativas ocupacionales ${ }^{4,18}$.

Con respecto al sexo, si bien se presentaron más mujeres que hombres con test positivo para síntomas de ansiedad y depresión, estas diferencias no fueron estadísticamente significativas, en contra de lo encontrado por autores nacionales ${ }^{15,16,19}$ e internacionales ${ }^{9,20}$. Sobre el particular en la literatura se han identificado un conjunto de factores que pueden explicar el predominio femenino en los trastornos de ansiedad y depresión, así como las diferencias entre los sexos de la presencia de otros trastornos psiquiátricos ${ }^{21}$, como la influencia de factores socioculturales (mayor disposición y facilidad de la mujeres a reportar y admitir sentimientos depresivos y ansiosos y la multiplicidad de roles en la familia) ${ }^{9,19,20}$, biológicos (predisposición genética, hormonas sexuales, reactividad endocrina frente al estrés, sistemas de neurotransmisión y determinantes neuropsicológicos) ${ }^{20}$ y la forma de 


\begin{tabular}{|c|c|c|c|c|c|c|}
\hline & \multicolumn{2}{|c|}{ Ansiedad } & \multicolumn{2}{|c|}{ Análisis bivariable } & \multicolumn{2}{|c|}{$\begin{array}{c}\text { Análisis } \\
\text { multivariable }\end{array}$} \\
\hline & Sí (\%) & No (\%) & OR & IC95\% & OR & IC95\% \\
\hline \multicolumn{7}{|l|}{ Sexo } \\
\hline Femenino & $435(75)$ & $145(25)$ & 0,86 & $0,6-1,1$ & & \\
\hline Masculino (Ref.) & $305(77,6)$ & $88(22,4)$ & 1 & & & \\
\hline \multicolumn{7}{|l|}{ Edad (ciclo vital) } \\
\hline Adultos jóvenes & $465(75,7)$ & $149(24,2)$ & 0,9 & $0,7-1,2$ & & \\
\hline Adolescentes (Ref.) & $275(76,6)$ & $84(25,4)$ & 1 & & & \\
\hline \multicolumn{7}{|l|}{ Programa académico } \\
\hline Área de la salud & $583(76,2)$ & $183(23,8)$ & 1,01 & $0,7-1,4$ & & \\
\hline Otros (Ref.) & $157(75,8)$ & $50(24,2)$ & 1 & & & \\
\hline \multicolumn{7}{|l|}{ Dificultades económicas } \\
\hline Sí & $377(75,8)$ & $90(19,3)$ & $1,6^{\star}$ & $1,2-2,2$ & $1,6^{\star}$ & $1,2-2,2$ \\
\hline No (Ref.) & $363(71,8)$ & $143(28,2)$ & 1 & & & \\
\hline \multicolumn{7}{|l|}{ Tiempo para descansar } \\
\hline Sí & $306(77,8)$ & $87(22,1)$ & 1,17 & $0,86-1,58$ & & \\
\hline No (Ref.) & $435(75,0)$ & $145(25,0)$ & 1 & & & \\
\hline \multicolumn{7}{|c|}{ Rendimiento académico acumulado } \\
\hline Deficiente & $3(50,0)$ & $3(50,0)$ & 0,3 & $0,006-1,5$ & & \\
\hline Aceptable / Bueno (Ref.) & $737(76,2)$ & $230(23,7)$ & 1 & & & \\
\hline \multicolumn{7}{|l|}{ Disfunción familiar } \\
\hline Sí & $475(76,3)$ & $147(23,6)$ & 1,05 & $0,7-1,4$ & & \\
\hline No (Ref.) & $265(75,5)$ & $86(24,5)$ & 1 & & & \\
\hline \multicolumn{7}{|l|}{ Soporte de amigos } \\
\hline Sí & $56(77,7)$ & $16(22,2)$ & 1,1 & $0,6-1,9$ & & \\
\hline No (Ref.) & $684(76,0)$ & $86(24,5)$ & 1 & & & \\
\hline \multicolumn{7}{|l|}{ Problemas familiares } \\
\hline Sí & $367(79,1)$ & $97(20,9)$ & $1,3 \dagger$ & $1,03-1,8$ & & \\
\hline No (Ref.) & $373(73,2)$ & $136(25,1)$ & 1 & & & \\
\hline \multicolumn{7}{|l|}{ Problemas con la pareja } \\
\hline Sí & $247(76,7)$ & $75(23,2)$ & 1,1 & $0,7-1,6$ & & \\
\hline No (Ref.) & $493(75,7)$ & $158(24,2)$ & 1 & & & \\
\hline \multicolumn{7}{|c|}{ Consumo habitual de alcohol } \\
\hline Sí & $508(80,1)$ & $126(19,8)$ & $1,8 \ddagger$ & $1,3-2,5$ & $1,8 \ddagger$ & $1,3-2,5$ \\
\hline No (Ref.) & $232(68,4)$ & $107(31,5)$ & 1 & & & \\
\hline \multicolumn{7}{|c|}{ Consumo de riesgo, perjudicial y riesgo de dependencia } \\
\hline Sí & $140(77,7)$ & $40(22,2)$ & 1,1 & $0,7-1,6$ & & \\
\hline No (Ref.) & $600(75,6)$ & $193(24,3)$ & 1 & & & \\
\hline \multicolumn{7}{|c|}{ Tratamiento psiquiátrico o psicológico } \\
\hline Sí & $114(77,5)$ & $33(22,4)$ & 1,05 & $0,7-1,4$ & & \\
\hline No (Ref.) & $626(75,7)$ & $158(24,2)$ & 1 & & & \\
\hline \multicolumn{7}{|c|}{ Antecedentes familiares de ansiedad o depresión } \\
\hline Sí & $114(79,7)$ & $29(20,2)$ & 1,2 & $0,8-1,6$ & & \\
\hline No (Ref.) & $313(74,6)$ & $88(21,9)$ & 1 & & & \\
\hline
\end{tabular}

Tabla 3. Asociación entre sintomatología de ansiedad con factores sociodemográficos, académicos, familiares y conductuales en estudiantes universitarios de Cartagena (Colombia), 2012. Ref.= categoría de referencia; a Significancia del modelo; OR ajustado por regresión $\left(p=0,02 ; \chi^{2}=27,1\right) * p=0,001, \uparrow p=0,003, \neq p=0,000$ 
expresión de los trastornos depresivos en los hombres $^{16,20}$. Sin embargo, existe acuerdo en considerar que los factores psicosociales y socioculturales no pueden explicar todas la diferencias entre ambos sexos, y dada la concordancia en los hallazgos en los diferentes grupos culturales, se podría concluir que las diferencias de sexo en las incidencias de los trastornos psiquiátricos son en gran parte psicobiológicas ${ }^{21}$. De allí que Dalla y Shors resalten la importancia de reconocer cuáles de los factores explicativos de las diferencias entre los sexos son de naturaleza biológica y cuáles de naturaleza social y, por ende, cuáles son inmutables y cuáles $\mathrm{no}^{21,22}$.

Dentro de los factores que se asociaron con la sintomatología de ansiedad y depresión estuvieron las dificultades económicas y problemas familiares; estos dos factores se relacionan entre sí, toda vez que las privaciones económicas generan estrés, que a su vez crea un ambiente emocional desfavorable en el hogar, percibiendo los adolescentes un ambiente negativo, caracterizado por carencia de recursos básicos para su desarrollo; esta dificultad disminuye la capacidad de los padres para proveer apoyo económico y estimulación cognitiva y emocional a sus hijos, limitando el ajuste adecuado ante las actividades y demandas, reduciendo las oportunidades de interacción social satisfactoria y adquisición de habilidades para el adecuado enfrentamiento a situaciones problemáticas, las cuales se han asociado como un elemento estructural en la relación con pares y la presencia de depresión ${ }^{4,23,24}$. A su vez, las condiciones de privación económica o pobreza pueden favorecer a la aparición de depresión en los padres, ocasionando conflictos maritales y problemas con los miembros de la familia ${ }^{24}$. La familia es el centro primario de socialización del individuo, en el cual se exponen los modelos a seguir a través de la observación constante del comportamiento de los padres y formas de relación social establecidas por el grupo familiar con los otros grupos. El apoyo familiar implica entonces un intercambio recíproco y voluntario de recursos, habilidades y servicios para un beneficio mutuo; cuando la demanda de apoyo familiar, ya sea de tipo económico, de cooperación o de afecto, supera las posibilidades que ofrece el grupo familiar, el joven puede volverse vulnerable ante las situaciones que le exigen esfuerzo y se le dificulta aceptar los retos, su estado de ánimo puede deprimirse y afectar su desempeño social o académico ${ }^{4,24}$, situación que es consecuente con los resultados de este estudio y los resultados de Álvarez et al., quienes indican que ciertos elementos del clima familiar, la relación entre padres e hijos y los recursos afectivos dentro del sistema familiar, están relacionados con la sintomatología depresiva en adolescentes ${ }^{25}$.

De igual forma, el poseer antecedentes familiares de ansiedad o depresión se asoció con el riesgo de sufrir de trastornos depresivos en los jóvenes, resultado coincidente con otros estudios que demuestran esta asociación ${ }^{15,24}$; Téllez et al. ${ }^{26}$ afirman que algunas formas de depresión son genéticas, lo que indica que alguna vulnerabilidad biológica pueda heredarse. Sin embargo, en este estudio también se pudo observar que estos síntomas se presentaron en personas que no tienen antecedentes de depresión, pudiendo sugerir que la variabilidad genética no es un factor determinante en la aparición de la misma. Ahora bien, con respecto a los antecedentes familiares de depresión, los parentescos más relacionados corresponden a los padres; Schramm et al. observaron que el trastorno aparece en los parientes de primer grado de pacientes depresivos con una frecuencia de 1,5 a 3 veces mayor que en la población general ${ }^{27}$. A su vez, Gelfand y Teti afirman que, particularmente cuando el miembro de la familia afectado es la madre, esta asociación puede deberse a predisposición genética o a otros comportamientos maternales que frecuentemente acompañan los estados depresivos como inactividad, inconsistencia en la disciplina e intromisión ${ }^{24,28}$.

En este estudio el factor que más se asoció con la presencia de sintomatología depresiva fue la presencia de síntomas de ansiedad. Watson et al. concluyeron que numerosos estudios han demostrado que las escalas de auto-reporte de ansiedad y depresión están altamente correlacionadas, sugiriendo de esta manera que evalúan el mismo constructo. Además, afirman que los síntomas ansiosos y depresivos co-ocurren en la mayoría de los pacientes y que la mitad de las personas con diagnóstico de ansiedad o depresión presentan co-morbilidad en cuanto al síndrome clínico completo $^{29}$. Otros estudios reportan la estrecha relación entre ansiedad y depresión, fundamentalmente en el análisis de las bases fisiológicas común de las mismas, las cuales se sustentan en investigaciones que ha encontrado que la respuesta de ansiedad y depresión comparten características funcionales como hiperactividad de la amígdala y un déficit de control del córtex pre-frontal; los neurotransmisores implicados y los antidepresivos utilizados en el tratamiento parecen ser los mismos de la respuesta de ansiedad y depresión ${ }^{30}$.

En razón al diseño de estudio y los criterios de determinación de la ansiedad y depresión, este es- 


\begin{tabular}{|c|c|c|c|c|c|c|}
\hline & \multicolumn{2}{|c|}{ Depresión } & \multicolumn{2}{|c|}{ Análisis bivariable } & \multicolumn{2}{|c|}{$\begin{array}{c}\text { Análisis } \\
\text { multivariablea }\end{array}$} \\
\hline & Sí (\%) & No $(\%)$ & OR & IC95\% & OR & IC95\% \\
\hline $\begin{array}{l}\text { Sexo } \\
\text { Femenino } \\
\text { Masculino (Ref.) }\end{array}$ & $\begin{array}{l}439(75,3) \\
295(75,7)\end{array}$ & $\begin{array}{c}152(24,7) \\
87(24,2)\end{array}$ & $\begin{array}{c}0,9 \\
1\end{array}$ & $0,6-1,2$ & & \\
\hline $\begin{array}{l}\text { Edad (ciclo vital) } \\
\text { Adultos jóvenes } \\
\text { Adolescentes (Ref.) }\end{array}$ & $\begin{array}{l}272(75,7) \\
462(75,5)\end{array}$ & $\begin{array}{c}87(24,3) \\
152(24,7)\end{array}$ & $\begin{array}{c}0,9 \\
1\end{array}$ & $0,7-1,3$ & & \\
\hline $\begin{array}{l}\text { Programa académico } \\
\text { Área de la salud } \\
\text { Otros (Ref.) }\end{array}$ & $\begin{array}{l}575(75,0) \\
159(76,8)\end{array}$ & $\begin{array}{c}191(24,9) \\
48(23,1)\end{array}$ & $\begin{array}{c}0,9 \\
1\end{array}$ & $0,6-1,3$ & & \\
\hline $\begin{array}{l}\text { Dificultades económicas } \\
\text { Sí } \\
\text { No (Ref.) }\end{array}$ & $\begin{array}{l}368(78,8) \\
366(72,3)\end{array}$ & $\begin{array}{c}99(21,2) \\
140(27,7)\end{array}$ & $\begin{array}{c}1,4^{*} \\
1\end{array}$ & $1,05-1,9$ & 1,49 & $1,0-2,0$ \\
\hline $\begin{array}{l}\text { Tiempo para descansar } \\
\text { Sí } \\
\text { No (Ref.) }\end{array}$ & $\begin{array}{l}438(75,5) \\
296(75,3)\end{array}$ & $\begin{array}{c}142(24,4) \\
97(24,6)\end{array}$ & $\begin{array}{c}1,01 \\
1\end{array}$ & $0,8-1,4$ & & \\
\hline $\begin{array}{l}\text { Rendimiento académico } \\
\text { Deficiente } \\
\text { Aceptable / Bueno (Ref.) }\end{array}$ & $\begin{array}{l}\text { umulado } \\
5(83,0) \\
729(75,3)\end{array}$ & $\begin{array}{c}1(16,6) \\
238(24,6)\end{array}$ & $\begin{array}{c}1,6 \\
1\end{array}$ & $0,1-1,4$ & & \\
\hline $\begin{array}{l}\text { Disfunción familiar } \\
\text { Sí } \\
\text { No (Ref.) }\end{array}$ & $\begin{array}{l}475(76,4) \\
259(73,7)\end{array}$ & $\begin{array}{c}147(23,6) \\
92(26,3)\end{array}$ & $\begin{array}{c}1,1 \\
1\end{array}$ & $0,8-1,5$ & & \\
\hline $\begin{array}{l}\text { Soporte de amigos } \\
\text { Sí } \\
\text { No (Ref.) }\end{array}$ & $\begin{array}{c}57(79,1) \\
677(75,1)\end{array}$ & $\begin{array}{c}15(20,2) \\
224(24,8)\end{array}$ & $\begin{array}{c}1,2 \\
1\end{array}$ & $0,6-2,2$ & & \\
\hline $\begin{array}{l}\text { Problemas familiares } \\
\text { Sí } \\
\text { No (Ref.) }\end{array}$ & $\begin{array}{l}370(79,7) \\
364(71,5)\end{array}$ & $\begin{array}{c}94(20,3) \\
145(28,5)\end{array}$ & $\begin{array}{c}1,5 \dagger \\
1\end{array}$ & $1,1-2,1$ & & \\
\hline $\begin{array}{l}\text { Problemas con la pareja } \\
\text { Sí } \\
\text { No (Ref.) }\end{array}$ & $\begin{array}{l}252(78,3) \\
482(74,0)\end{array}$ & $\begin{array}{c}70(21,7) \\
169(26,0)\end{array}$ & $\begin{array}{c}1,2 \\
1\end{array}$ & $0,9-1,7$ & & \\
\hline $\begin{array}{l}\text { Consumo habitual de alcc } \\
\text { Sí } \\
\text { No (Ref.) }\end{array}$ & $\begin{array}{l}\text { 1ol } \\
486(76,8) \\
248(73,1)\end{array}$ & $\begin{array}{c}148(23,3) \\
91(26,8)\end{array}$ & $\begin{array}{c}1,2 \\
1\end{array}$ & $0,8-1,6$ & & \\
\hline $\begin{array}{l}\text { Consumo de riesgo, perju } \\
\text { Sí } \\
\text { No (Ref.) }\end{array}$ & $\begin{array}{l}\text { licial y riesg } \\
133(20,5) \\
601(75,7)\end{array}$ & $\begin{array}{l}\text { le depende } \\
47(26,1) \\
192(24,2)\end{array}$ & $\begin{array}{c}0,9 \\
1\end{array}$ & $0,6-1,3$ & & \\
\hline $\begin{array}{l}\text { Tratamiento psiquiátrico } \\
\text { Sí } \\
\text { No (Ref.) }\end{array}$ & $\begin{array}{l}\text { psicológico } \\
117(79,5) \\
617(74,7)\end{array}$ & $\begin{array}{c}30(20,5) \\
209(25,3)\end{array}$ & $\begin{array}{c}1,3 \\
1\end{array}$ & $0,8-1,2$ & & \\
\hline $\begin{array}{l}\text { Ansiedad } \\
\text { Sí } \\
\text { No (Ref.) }\end{array}$ & $\begin{array}{c}654(88,2) \\
80(34,4)\end{array}$ & $\begin{array}{l}351(27,1) \\
204(24,1)\end{array}$ & $\begin{array}{c}14,2 \ddagger \\
1\end{array}$ & $10,0-20,2$ & $13,9 \ddagger$ & $9,7-19,7$ \\
\hline $\begin{array}{l}\text { Antecedentes familiares c } \\
\text { Sí } \\
\text { No (Ref.) }\end{array}$ & $\begin{array}{l}\text { ansiedad c } \\
316(78,8) \\
418(73,0)\end{array}$ & $\begin{array}{l}\text { epresión } \\
85(21,2) \\
154(26,0)\end{array}$ & $\begin{array}{c}1,3 \S \\
1\end{array}$ & $1,01-1,8$ & 1,2 & $0,8-1,8$ \\
\hline
\end{tabular}

Tabla 4. Asociación entre sintomatología de depresión con factores sociodemográficos, académicos, familiares y conductuales en estudiantes universitarios de Cartagena (Colombia), 2012. Ref.= categoría de referencia; a Significancia del modelo; OR ajustado por regresión $\left(p=0,000 ; \chi^{2}=254,6\right){ }^{*} p=0,01, \uparrow p=0,003, \neq p=0,000, \S p=0,04, \uparrow p=0,04$ 
tudio no permite dar un diagnóstico definitivo de trastornos de ansiedad y depresión, sino su exploración a partir de su sintomatología, pues el cuestionario de Goldberg solo se utiliza para cribado. Gracias a la utilización de procedimientos, métodos y escalas de valoración válidos y confiables se garantiza la utilización de esta información en el contexto en el que fueron aplicados, permitiendo hacer una aproximación a la problemática de salud mental de los jóvenes universitarios de Cartagena, siendo utilizados en pro del mejoramiento y bienestar psicosocial de sus educandos y de los profesionales en formación. Sin embargo, dada la alta prevalencia es preciso hacer un estudio más exhaustivo, el cual incluya entrevistas psiquiátricas para determinar el diagnóstico de ansiedad y depresión y diferenciarlo así de un posible estado de desmoralización, el cual comparte características con la depresión.

\section{AGRADECIMIENTOS}

Los autores y el grupo de Salud Pública de la Facultad de Odontología de la Universidad de Cartagena (GISPOUC) expresan su agradecimiento a los estudiantes que participaron en el estudio y a Carlos Acosta y Elisa Álvarez por su apoyo en el proceso de recolección de la información.

\section{BIBLIOGRAFÍA}

1. Kastrup MC, Ramos AB. Global Mental Health. Dan Med Bull.2007; 54 (1): 42-3.

2. Sánchez R, Cáceres H, Gómez D. Ideación suicida en adolescentes universitarios: Incidencia y factores asociados. Biomedica. 2002; 22: 407-16.

3. Arco G, López S, Heilborn V, Fernández F. Terapia breve en estudiantes universitarios con problemas de rendimiento académico y ansiedad: Eficacia del modelo "La Cartuja". Int J Clin Health Psychol. 2005; 5 (3): 589-608.

4. Pardo G, Sandoval A, Umbarila D. Adolescencia y depresión. Rev Colomb Psiquiatr. 2004; 13: 13-28.

5. Blum R. Un modelo conceptual de salud del adolescente. En: Dulanto, edit. El Adolescente. México: Mc Graw Hill; 2000. p. 656-72.

6. Lewinsohn P, Rohde P, Seeley J, Klein D, Gotlib I. Natural course of adolescent major depressive disorder in a community sample: Predictors of recurrence in young adults. Am J Psychiatry. 2000; 157 (10): 1584-97.

7. Aalto-Setala T, Marttunen M, Tuulio-Henriksson A, Poikolainen K, Lonnqvist J. Depressive symptoms in adolescence as predictors' early adulthood depressive disorder and maladjustment. Am J Psychiatry. 2002; 152: 1235-7.

8. Santacruz C, Gómez R, Posada V, Viracachá P. A proposito de un analisis secundario de la encuesta nacional de salud mental y consumo de sustancias psicoactivas. 1995. Bogotá: Ministerio de Salud- Pontificia Universidad Javeriana.

9. Balanza S, Morales Moreno I, Guerrero Muñoz J. Prevalen- cia de ansiedad y depresión en una población de estudiantes universitarios: factores académicos y socio-familiares asociados. Clínica y Salud. 2009; 20 (2): 177-87.

10. Villamil V, Valencia M, Medina ME, Juárez García F. Validación de la Prueba de Identificación de Trastornos Derivados del Consumo de Alcohol (AUDIT) en pacientes mexicanos con esquizofrenia. Rev Panam Salud Publica. 2009; 26 (4): 283-9.

11. Arrieta K. Consumo patológico de alcohol entre los estudiantes de la universidad de Cartagena, 2008. Rev Salud Pública. 2009; 11 (6): 878-86.

12. Smilkstein G. The Family Apgar: A proposal for a family function test and its use by physicians. J Fam Pract. 1978; 6: 1231-9.

13. Goldberg D, Bridges K, Duncan-Jones P, Grayson D. Detecting anxiety and depression in general medical settings. Br Med J. 1988; 297 (6653): 897-9.

14. Greenland S. Modeling and variable selection in epidemiologic analysis. Am J Public Health. 1989; 79: 340-9.

15. Arrivillaga M, Cortes C, Goicochea V, Lozano T. Caracterización de la depresión en jóvenes universitarios. Universitas Psicológica. 2004; 3 (1): 17-26.

16. Amézquita M, González R, Zuluaga D. Prevalencia de la depresión, ansiedad y comportamiento suicida en la población estudiantil de pregrado de la Universidad de Caldas, año 2000. Rev Colomb Psiquiatr. 2003; 32 (4): 341-56.

17. Campo G, Gutiérrez JC. Psicopatología en estudiantes universitarios de la Facultad de Salud, Univalle. Rev Colomb de Psiquiatr. 2001; 30: 351-8.

18. Gómez C, Rodríguez N. Factores de riesgo asociados al síndrome depresivo en población Colombiana. Rev Colomb. Psiquiatr. 1997; 26 (1): 23-5.

19. Guavita P, Sanabria P. Prevalencia de sintomatología depresiva en una población estudiantil de la Facultad de Medicina de la Universidad Militar Nueva Granada. Rev Fac Med Univ Nac Colomb. 2006; 54 (2): 76-85.

20. Dávila A, Ruiz R, Moncada L, Gallardo I. Niveles de ansiedad, depresión y percepción de apoyo social en estudiantes de odontología de la Universidad de Chile. Rev Psicol. 2011; 20 (2): 147-72.

21. Arenas MC, Pulgcerver A. Diferencias entre hombres y mujeres en los trastornos de ansiedad: una aproximación psicobiológica. Escritos de Psicología. 2009; 3 (1): 20-9.

22. Dalla C, Shors TJ. Sex differences in learning processes of classical and operant conditioning. Physiol Behav. 2009; 97: 229-38.

23. Hao L. Poverty, public assistance, and children in intact and single-mother families. J Fam Econ. 1995; 16: 181205.

24. Eamon MK. Influences and mediators of the effect of poverty on young adolescent with depressive symptoms. J Youth Adolesc. 2002; 31 (3): 231-42.

25. Álvarez $M$, Ramírez $B$, Silva A, Coffin N, Jiménez L. La relación entre depresión y conflictos familiares en adolescentes. Rev Int Psicol Ter Psicol. 2009; 9 (2): 205-16.

26. Téllez J. La noradrenalina: su rol en la depresión. Rev Colomb Psiquiatr. 2000; 29 (1): 50-73.

27. Schramm E. Psicoterapia Interpersonal. Barcelona: Masson; 1998.

28. Gelfand DM, Teti DM. The effects of maternal depression on children. Clin Psychol Rev. 1990; 10: 329-53.

29. Watson D, Clark LA, Carey G. Positive and negative affectivity and their relation to anxiety and depression. J Abnorm Psycho. 1988; 93 (3): 346-53.

30. Rahola JG, Micó JA. Ansiedad y depresión: ¿tan lejos o tan cerca? En: Gutiérrez M, Ezcurra J, Pichot P, edits. Ciclo vital y trastornos psiquiátricos graves. Madrid: Aula Medica; 2002. p. 483-530. 